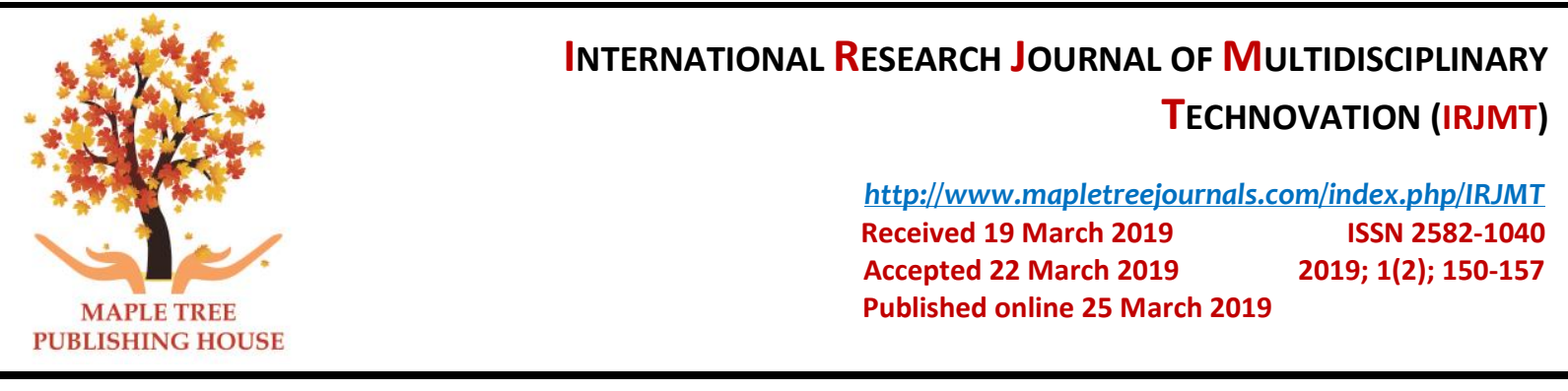

\title{
Implementation of Differential Algorithm for Busbar Protection
} Bharath Kumar Sugumar ${ }^{1 *}$,Sujatha Balaraman ${ }^{2}$

${ }^{1} P G$ Scholar, Department of Electrical Engineering, Government College of Technology, Coimbatore, TN, India

${ }^{2}$ Associated Professor, Department of Electrical Engg, Government College of Tech, Coimbatore, TN, India

*Corresponding author E-Mail ID: sbharathkumarukl@gmail.com, Mobile: +91 7373322312

DOI: https://doi.org/10.34256/irjmt19220

\begin{abstract}
Faults in power systems are classified as internal and external faults. Faults within the zone are termed as internal faults whereas; the faults outside the Zone are called as external faults. Ideally, a relay outward after the protection of a zone should operate only for internal faults. It should restrain from operating for external faults or through faults. In this project, the busbar protection using differential protection scheme has been investigated for internal and external faults. The current magnitude from the Current Transformer is compared with a preset value and when the current exceeds the preset value, and then a trip command is given to associated circuit breaker. In this work, an algorithm has been developed to improve the selectivity of the relay and the same is tested on three-phase bus bar having two incoming lines and three outgoing lines at different fault levels and the results are verified for internal and external faults. The entire algorithm is programmed and graphical views of relay performance are verified using the MP LAB platform.
\end{abstract}

Keywords: Busbar protection, CT saturation, Ratio mismatch of CT secondary. Works on busbar protection, Numerical relay.

\section{INTRODUCTION}

The relay used in power system protection are of differential types. Among them differential relay is very usually used relay for protecting transformers and generators from localized faults. Differential relays are hypersensitive to the faults occurred within the zone. Most of the relays operate when any quality exceeds before a predetermined value for example over current relay operates when current through it compare predetermined value. But the principle of differential relay is rather different. It operates depending upon the difference between two or more similar. A differential relay is defined as the relay that operates when the phase difference of two or more identical electrical quantities exceeds a predetermined amount. The differential relay works on the principle of comparison between the point and magnitude of 2 or additional equivalent electrical quantities. Comparing 2 electrical quantities in a very circuit victimization differential relays is easy in application and positive in action.

For benchmark, consider the comparison of the current entering a protected line and the current leaving it. If this enters the protected line is quite this leaves it, then the extra current must flow in the fault. The distinction between the 2 electrical quantities will operate a relay to 
isolate the circuit. For the operation of the differential relay, it should have two or more electrical magnitude, and these magnitude should have a phase displacement . Any types of the relay can operate as a differential relay depends on upon the way it is connected in a circuit. In alternative words, it doesn't depend on the construction of the relay it depends on the way it is connected to the circuit.

Differential protection provides unit protection. The protected zone is strictly famous by the situation of current and potential transformers. The phase difference is achieved by suitable connections of secondary of CTs and PTs. The differential protection principle is utilized for the protection of generator, generator-transformer units, transformers, feeders, giant motors, and bus-bars.

\section{BRIEF EXPLANATION OF RELAY}

\subsection{Over View of Relay}

A relay is an electrically operated switch. Many relays use AN magnet to automatically operated the switch, however alternative in operation principles are used, like solid-state relays. Relays are used whether it's necessary to regulate a circuit by a separate low-power signal, or wherever many circuits should be controlled by one signal. The first relays were exhaust in long distance telegraph circuits like amplifiers, they extended the signal coming back in from one circuit and re-transmitted it on another circuit. Relays were used extensively in phone exchanges and early computers to perform logical operations. A type of relay that will handle the high power needed to directly manage an electrical motor or alternative hundreds are termed a contactor. Relays management power circuits with no moving elements, instead of employing a semiconductor unit to perform the shift. Relays with graduated in operation characteristics and generally multiple in operation coils are accustomed defend electrical circuits from overload or faults; in fashionable wattage systems these functions are performed by digital instruments still called "protective relays".

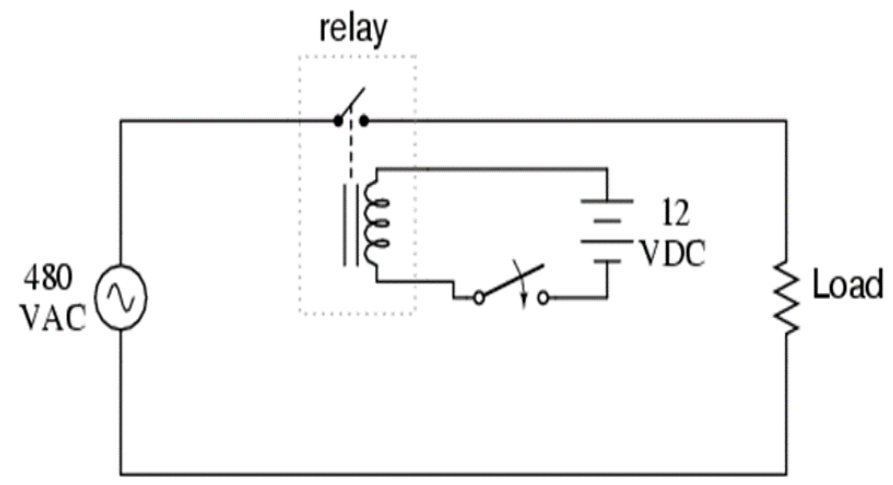

Fig 1. Circuit Diagram of Relay

\subsection{Overcurrent Relay}

It finds its application from the fact that in the event of a fault the current will increase to a value several times greater than maximum load current. A relay that operates or picks up once its current exceeds a preset price (setting value) is termed Over-current relay. Over-current protection protects power systems against excessive currents that square measure caused by short circuits. Ground faults, etc. Over-current relays are often accustomed shield much any power grid parts, i.e. transmission lines, transformers, generators, or motors. For feeder protection, there would be over one over-current relay to safeguard totally different sections of 
the feeder. These over-current relays need to coordinate with each such that the relay nearest fault operates first.

\subsection{Inverse Definite Minimum Time}

The inverse definite minimum time (IDMT) protecting relays were developed to beat the shortcomings of the Definite Time Overcurrent Relays. If the supply electric resistance remains constant and also the fault current changes appreciably as we tend to move aloof from the relay then it's advantageous to use IDMT overcurrent protection to achieve high-speed protection over an outsized section of the protected circuit. However, if the supply electric resistance is considerably larger than the feeder electric resistance then the characteristic of the IDMT relay can't be exploited and DTOC could also be used. Secondly, if the supply electric resistance varies and becomes weaker with less generation throughout lightweight masses then this results in slower clearance time thence negating the aim of the IDMT relay.IEC normal 60255-151 specifies the IDMT relay curves as shown below. The four curves in Table one square measure derived from the currently withdrawn British normal bachelor's degree 142 . The other 5, are derived from the ANSI standard C37.112.



Fig2. IDMT Relay Curve

While it's a lot of common to use IDMT relays for current protection it's doable to utilize IDMT mode of operation for voltage protection. It is possible to program customized curves in some protective relays and other manufacturers have special curves specific to their relays. Some numerical relays will be accustomed to offer inverse time overvoltage protection or negative sequence overcurrent protection.

\section{PROTECTION OF BUSBAR}

\subsection{Blocking Logic In O\C Relay}

After limitations experienced with the use of black up over-current relays, dedicated busbar protection using numerical over-current relays with instantaneous protection and blocking logic are used to give a cost-effective and reliable result. This can also provide a discriminative phase and earth fault protection. The logic can be described as when there is a through the fault in any feeder, it will block the income to operate but when there is a busbar fault, the incomer breaker will operate. A short time delay is applied to the incomer breaker to receive a block signal from the feeder relay. For non-directional relay greater than $40 \mathrm{~ms}$ setting is found satisfactory and more than $60 \mathrm{~ms}$ for the directional relay. This delay takes into account CT saturation. 
For sectionalized busbars, 2 settings square measure to be incorporated wherever the best setting can trip the section or mechanical device breaker and once a time delay, if the fault current still persists, the sections will monitor individually and isolate the faulty part. The scheme got an inherent advantage that whenever there is the failure of the relay of the outgoing feeder; the busbar scheme will operate within a short period of time instead of the delayed operation of incomer backup overcurrent protection.

\subsection{Selection of CT Ratios}

The correct method of setting the CT ratios for the busbar differential protection. It can be seen that the CT ratios of all the CTs are equal and are based on the primary current of that feeder which carries the maximum current. Thus, all the CT ratios are 3000/1 A. There is no spill current through the OC relay connected within the spill path and also the theme remains stable.

\subsection{Internal Fault}

The fault shifts by a small distance to the left and becomes an internal fault, still drawing the same current, the situation dramatically changes as far as b3, b4 \& b5 is concerned. This is depicted wherein it can be seen that b3, b4 \& b5 now does not carry any fault current. Since b1 and b2 are not carrying excessive primary currents, they transform the current without too much error. There is thus a spill current in the spill path and the scheme operates as expected.



Fig3. Behaviour of busbar differential scheme on internal fault

\subsection{External Fault}

An external fault beyond b3, b4 \& b5 (receiving end current). It can be seen that b3, b4 \& $\mathrm{b} 5$, the CT on the faulted feeder, has to carry the sum of all currents fed into fault by various feeders. Therefore, receiving end current sees a substantially larger primary current than either b1 or b2. In all likelihood, receiving end current transformer will, therefore, become saturated. We can, therefore, no longer assume that receiving end current transformer will faithfully transform the fault current. For the sake of illustration, we have assumed that the secondary current of receiving end current transformer is only 1.333A instead of $1.333 \mathrm{~A}$ It can be seen from fig 3.6 that this results into a spill current of 1.333 A causing the scheme to maloperation, i.e. lose stability on the external fault. 
In the worst case scenario, b1 and b2 continue to transform faithfully as per their nameplate $\mathrm{CT}$ ratio but receiving end current transformer, which carries the total fault current, gets completely saturated. This clearly indicates the occurrence of an imbalance in the transformer secondary current, resulting in substantial spill current. This situation most likely will cause the scheme to operate. The operation of a differential scheme under external faults is, therefore, clearly a case of maloperation.



Fig4. Behaviour of busbar differential scheme on external fault

\section{MPLAB Platform}

MPLAB X IDE could be a computer code program that runs on a laptop (Window, Mac OS, Linux) to develop the applying for chip microcontrollers and digital signal controllers. It is known as an associate Integrated Development atmosphere (IDE) as a result of it provides one integrated "environment" to develop code for embedded microcontrollers.

MPLAB X Integrated Development atmosphere brings a bunch of options to reinforce the correct expertise throughout the planning section of your project. Based on the open source NetBeans IDE from Oracle, Microchip is able to add many frequencies requested features very quickly and easily, while also providing a much extensible architecture to bring you, even more, features fast - including added support for AVR devices.

\section{RESULT \& DISCUSSION}

\subsection{CASE 1- Normal Operation of Busbar Differentia Protection}

In three phase differential relay for normal operation, the net incoming current value and outgoing current value are same in each phase.

\subsection{CASE 2- Busbar Differential Protection Under External Fault}

Suppose an external fault occurs beyond the protection zone, say at h, i \& j. The CT on the faulted feeder has to carry the sum of all currents fed into fault by various feeders. Therefore, receiving end current sees a substantially larger primary current than either $f$ or $g$. In all likelihood, receiving end current transformer will, therefore, become unsaturated. We can, therefore, no longer assume that receiving end current transformer will faithfully transform the fault current. For the sake of illustration, we have assumed that the secondary current of 

receiving end current transformer is only $1.333 \mathrm{~A}$. It can be seen that this results into a spill current of 1.333 A causing the scheme to maloperation, i.e. lose stability on the external fault.

In the worst case scenario, $f$ and $g$ continue to transform faithfully as per their nameplate CT ratio but receiving end current transformer, which carries the total fault current, gets completely saturated. This clearly indicates the occurrence of an imbalance in the transformer secondary current, resulting in substantial spill current. This situation possibly can cause the theme to control. The operation of a differential scheme under external faults is, therefore, clearly a case of maloperation.

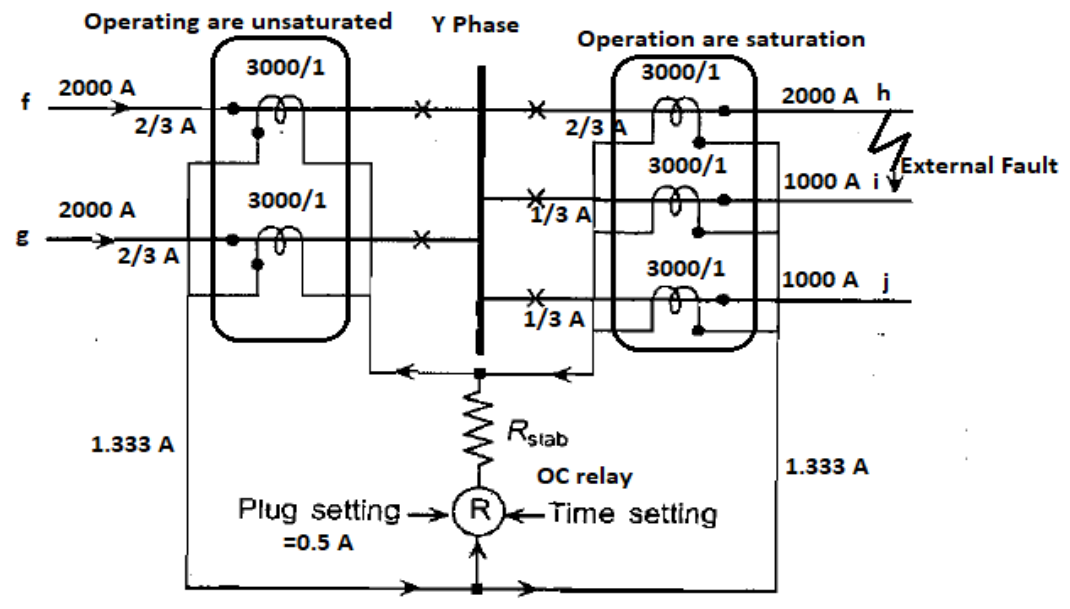

Fig5. Behaviour of busbar differential scheme on external fault

\subsection{CASE 3- Internal Fault of Busbar Differential Protection}

The fault shifts by a small distance to the left and becomes an internal fault, still drawing the same current, the situation dramatically changes as far as $\mathrm{h}, \mathrm{i} \& \mathrm{j}$ is concerned. This is depicted wherein it can be seen that $\mathrm{h}, \mathrm{i} \& \mathrm{j}$ now does not carry any fault current. Since $\mathrm{f}$ and $\mathrm{g}$ are not carrying excessive primary currents, they transform the current without too much error. There is thus a spill current in the spill path and the scheme operates as expected.

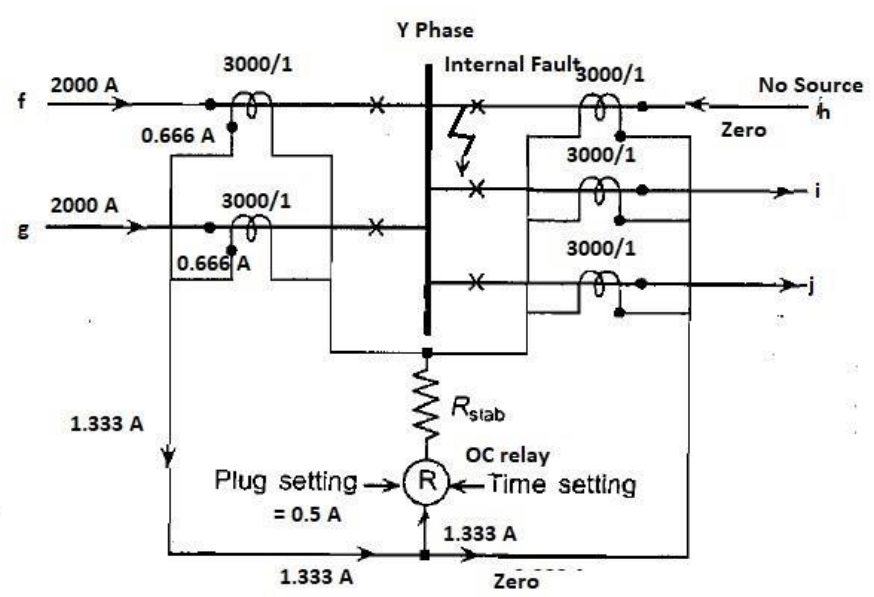

Fig6. Behaviour of busbar differential scheme on internal fault 
Table 1. Relay Characteristic

\begin{tabular}{|c|c|c|c|c|}
\hline Current & Idmt val & TMS & PSM & Restricted inverse \\
\hline 0.5 & 0.5 & 0.02 & 1 & 0.198019802 \\
\hline 0.55 & 0.5 & 0.02 & 1.1 & 0.163083766 \\
\hline 0.6 & 0.5 & 0.02 & 1.2 & 0.142180095 \\
\hline 0.65 & 0.5 & 0.02 & 1.3 & 0.128268377 \\
\hline 0.7 & 0.5 & 0.02 & 1.4 & 0.118343195 \\
\hline 0.75 & 0.5 & 0.02 & 1.5 & 0.11090573 \\
\hline 0.8 & 0.5 & 0.02 & 1.6 & 0.105124836 \\
\hline 0.85 & 0.5 & 0.02 & 1.7 & 0.100502513 \\
\hline 0.9 & 0.5 & 0.02 & 1.8 & 0.096722192 \\
\hline 1 & 0.5 & 0.02 & 2 & 0.090909091 \\
\hline 1.1 & 0.5 & 0.02 & 2.2 & 0.086648287 \\
\hline
\end{tabular}

$\begin{array}{llll}\text { Restricted Inverse } & = & \left((1 /(0.339(0.238 /(\mathrm{PSM}))))^{*}\right. & \text { TMS*1000.0 })^{*} 10-3 \\ \text { Current } & = & 1.333 \mathrm{~A} \\ \text { IDMTval } & = & 0.5 \\ \text { TMS } & = & 0.02 \\ \text { PSM } & = & \text { Current/IDMTval } \\ \text { PSM } & = & 1.333 / 0.5 \\ & = & 2.66 \\ \text { Restricted Inverse } & = & \left((1 /(0.339(0.238 /(\mathrm{PSM})))) * \mathrm{TMS}^{*} 1000.0\right)^{*} 10-3 \\ & = & ((1 /(0.339-(0.238 /(2.66)))) * 0.02 * 1000.0) * 10-3 \\ \text { Time } & = & 0.080152 \mathrm{sec}\end{array}$

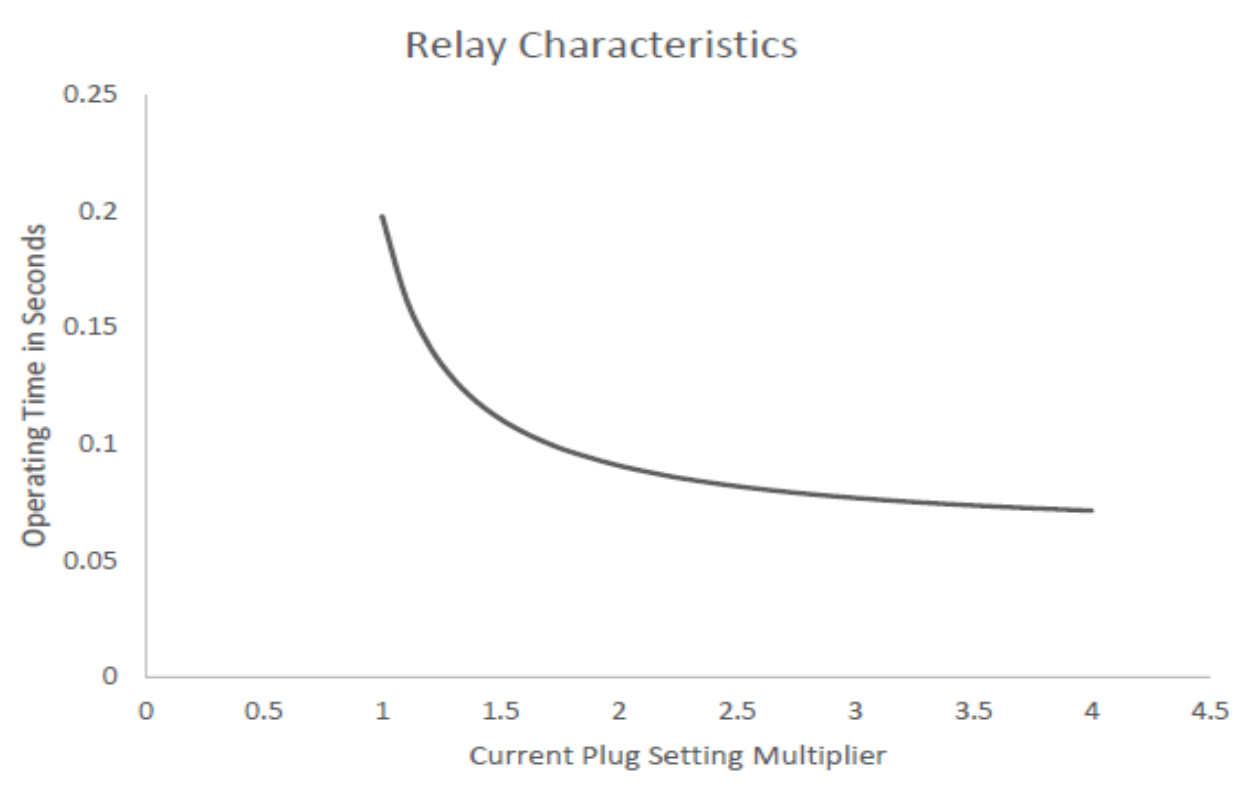

Fig7. Time - Current Characteristics for different Value of TMS 


\section{CONCLUSION}

In this project, a differential algorithm has been proposed for the protection of the busbar for internal and external faults. The algorithm gives a fast response, particularly for busbar differential protection. During an external fault, the relay remains stable since a blocking signal is received before the protection operates. During an internal fault, a trip command is issued and the protection relay operates before saturation is detected. The proposed scheme reduces the operating time of a relay on an internal fault.

\section{REFERENCES}

[1] Kleber M. Silva, Alfredo Miguel P. Escudero, Felipe V. Lopes and Flavio B. Costa, June 2018, “A wavelet - Based Busbar Differential Protection”, IEEE transactions on power delivery, Vol 33, No. 3, June 2018.

[2] Mohindar S.Sachdev, Life Fellow, S. Sidhu, Harjeet Singh Gill, July 2000, “A Busbar Protection Technique and Its Performance During CT Saturation and CT Ratio-Mismatch", IEEE transactions on power delivery, Vol. 15, No. 3, July 2000.

[3] Y.C. Kang, J.S. Yun, B.E. Lee, S.H. Kang, S.I. Jang and Y.G. Kim, "Busbar Differential Protection in Conjunction with a Current Transformer Compensating Algorithm", IET Generation Transmission Distribution ,2008, 2, (1),pp. 100-109.

[4] Yong-Cheol Kang, Ui-Jai Lim, Sang-Hee Kang, Peter A. Crossley, April 2005, “A Busbar Differential Protection Relay Suitable for Use with Measurement Type Current Transformer", IEEE transactions on power delivery, Vol. 20, No. 2, April 2005.

[5] Cesareo Fernandez, October 2001, "An Impedance- Based CT Saturation Detection Algorithm for Bus-bar Differential Protection”, IEEE transactions on power delivery, Vol. 16, No. 4, October 2001. 